\title{
A Spectrum of Modes of Knowledge Sharing between Agents
}

\author{
Alessio Lomuscio* and Mark Ryan ${ }^{\dagger}$ \\ * Department of Electronic Engineering, QMW College, London, UK \\ A.R.Lomusciodelec.qmw.ac.uk \\ $\dagger$ School of Computer Science, University of Birmingham, Birmingham, UK \\ M.D.Ryan@es.bham.ac.uk
}

\begin{abstract}
The logic $\mathrm{S} 5_{n}$ is widely used as the logic of knowledge for ideal agents in a multi-agent system. Some extensions of $\mathrm{S} 5_{n}$ have been proposed for expressing knowledge sharing between the agents, but no systematic exploration of the possibilities has taken place. In this paper we present a spectrum of degrees of knowledge sharing by examining and classifying axioms expressing the sharing. We present completeness results and a diagram showing the relations between some of the principal extensions of $\mathrm{S}_{2}$ and discuss their usefulness. The paper considers the case of a group of two agents of knowledge.
\end{abstract}

\section{Introduction}

The modal logic $\mathrm{S}_{n}$ (see for example $[18,17]$ ), whose mono-modal fragment $\mathrm{S} 5$ was first proposed in [7] to represent knowledge, has been used to model knowledge in multi-agent systems (MAS) for some years now [4]. The logic $\mathrm{S} 5_{n}$ is a classical modal logic containing $n$ modalities $\square_{i}$, where $i$ is in a set $A$ of agents, expressing the private knowledge of agent $i$. Results that extend the logic $\mathrm{S} 5_{n}$ to model group properties such as common knowledge and distributed knowledge within a group of agents are also well known $([4,17])$.

The logic $\mathrm{S} 5_{n}$ models an ideal set of agents, in particular agents enjoy positive and negative introspection and their knowledge is closed under implication; in other words they are perfect reasoners.

A peculiarity of the logic $\mathrm{S} 5_{n}$, is that there is no a priori relationship between the knowledge of the various agents. In some applications, however, this might not be what is desired. For example, a central processing unit $j$ of a collective map making ([3]) robotic MAS should be told of any knowledge acquired by any other agent. Therefore the agent $j$ should know everything that is known by any agent. In the formal language of modal logic, under the usual assumptions of ideality, this scenario can be represented by $\mathrm{S} 5_{n}$ enriched by the axiom:

$$
\square_{i} p \Rightarrow \square_{j} p, \text { for all } i \in A .
$$

Another example of knowledge sharing between agents concerns a MAS whose agents (databases in this example) have computation capabilities that can be ordered. If the 
agents are executing the same program on the same data then it is reasonable to model the MAS by enriching the logic $\mathrm{S} 5_{n}$ by:

$$
\square_{i} p \Rightarrow \square_{j} p ; i \prec j
$$

where $\prec$ expresses the order in the computational power at disposal of the agents. In these two cases, some information is being shared among the agents of the group.

A third example of sharing in the literature is the axiom

$$
\diamond_{i} \square_{j} p \Rightarrow \square_{j} \diamond_{i} p ; i \neq j
$$

[2] which says that: if agent $i$ considers possible that agent $j$ knows $p$ then agent $j$ must know that agent $i$ considers possible that $p$ is the case.

It is easy to imagine other meaningful axioms that express interactions between the agents in the system; clearly there is a spectrum of possible degrees of knowledge sharing. At one end of the spectrum is $\mathrm{S} 5_{n}$, with no sharing at all. At the other end, there is $\mathrm{S} 5_{n}$ together with

$$
\square_{i} p \Leftrightarrow \square_{j} p \text {; for all } i, j \in A,
$$

saying that the agents have precisely the same knowledge (total sharing). The three examples mentioned above exist somewhere in the (partially ordered) spectrum between these two extremes.

Some instances of such systems have already been identified in $[2,1,15]$ and in other papers. Our aim in this paper is to explore the spectrum systematically. We restrict our attention to the case of two agents (i.e. to extensions of $\mathbf{S} 5_{2}$ ), and explore axiom schemas of the forms

$$
\begin{aligned}
& \unlhd p \Rightarrow \square p \\
& \square p \Rightarrow \square \square p \\
& \triangleright \boxminus p \Rightarrow \boxminus p \\
& \bullet \boxminus p \Rightarrow \boxminus \boxminus p
\end{aligned}
$$

where each occurrence of $\square$ is in the set $\left\{\diamond_{1}, \square_{1}, \diamond_{2}, \square_{2}\right\}$.

Technically we will prove correspondence properties and completeness for extensions of $\mathrm{S}_{2}$ with axioms of these forms. Naturally, this will not give the complete picture: there may be interesting axioms of other forms than those listed above. However, analysis of the literature certainly suggests that most axioms studied for this purpose are of one of these forms. They are sufficient for expressing how knowledge and facts considered possible are related to each other up to a level of nesting of two, which is already significant for human intuition. Note also that the examples above are included in the axiom patterns.

The rest of this paper is organised as follows. In Section 1.1 we fix the notation and recall two known results that we will extensively use in the following. In Section 2 we analyse and discuss the interaction axiom schema the form $\square p \Rightarrow \square p$. We will then move to Section 3 where we discuss the case of the consequent composed by two modal operators. In Section 4 we will analyse the interaction axioms resulting from two nested modalities both in the antecedent and in the consequent. Finally in Section 5 we present the spectrum of interaction axioms that is generated. 


\subsection{Preliminaries}

Our syntax is the standard bi-modal language $\mathcal{L}$, defined from a set $P$ of propositional variables:

$$
\phi::=p|\neg \phi| \phi_{1} \wedge \phi_{2} \mid \square_{i} \phi
$$

where $p \in P, i \in\{1,2\}$.

As standard, we use bi-modal Kripke frames $F=\left(W, R_{1}, R_{2}\right)$ and models $M=$ $\left(W, R_{1}, R_{2}, \pi\right)([13])$ to interpret the language $\mathcal{L}$. Interpretation, satisfaction and validity are defined as standard (see for example [10]).

The paper is devoted to extensions of $\mathrm{S}_{2}$, which is defined by the following Hilbert style axioms and inference rules.

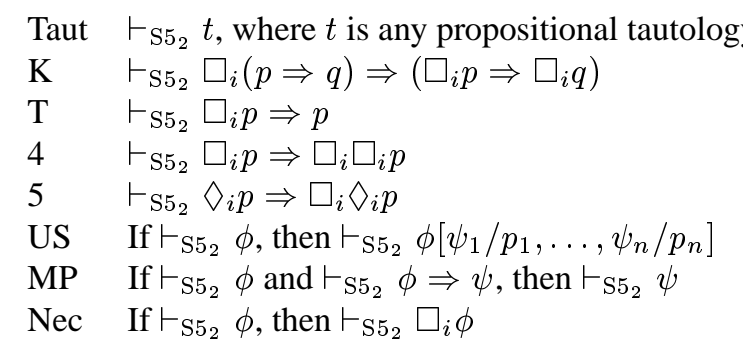

In the above the index $i$ is in $\{1,2\}$.

The symbol $\vdash$ means provability in that logic, or in the extension under consideration. By S $5_{2}+\{\phi\}$ we denote the extension of $\mathrm{S} 5_{2}$ in which the formula $\phi$ is added to the axioms.

The following is also widely known.

Theorem 1. The logic $S 5_{2}$ is sound and complete with respect to equivalence frames $F=\left(W, \sim_{1}, \sim_{2}\right)$.

We will always be working in the class $\mathcal{F}_{\mathcal{E}}$ of equivalence frames.

We also recall a standard lemma that we will use in this paper.

Lemma 1. For any $\phi \in \mathcal{L}$, we have $\vdash \square_{i} \phi \Leftrightarrow \square_{i} \square_{i} \phi \Leftrightarrow \searrow_{i} \square_{i} \phi$ and $\vdash \diamond_{i} \phi \Leftrightarrow$ $\square_{i} \diamond_{i} \phi \Leftrightarrow \diamond_{i} \diamond_{i} \phi$ where $i \in A$.

\section{Interaction axioms of the form $\sqcup p \Rightarrow \triangleright p$}

We start with extensions of $\mathrm{S}_{2}$ with respect to interaction axioms that can be expressed as:

$$
\triangleright \phi \Rightarrow \triangleright \phi, \text { where } \square \in\left\{\square_{1}, \square_{2}, \diamond_{1}, \diamond_{2}\right\} .
$$

There are 16 axioms of this form; factoring 1-2 symmetries reduces this number to 8 , of which 4 are already consequences of $\mathrm{S}_{2}$ and therefore do not generate proper extensions ${ }^{1}$. The remaining 4 are proper extensions of $\mathrm{S}_{2}$ and give rise to correspondence properties as described in Fig. 1.

\footnotetext{
${ }^{1}$ The four are $\square_{1} p \Rightarrow \square_{1} p, \square_{1} p \Rightarrow \diamond_{1} p, \square_{1} p \Rightarrow \diamond_{2} p$, and $\diamond_{1} p \Rightarrow \diamond_{1} p$.
} 


\begin{tabular}{|c|c|}
\hline Interaction Axioms & Completeness \\
\hline$\square_{1} p \Rightarrow \square_{2} p$ & $\sim_{2} \subseteq \sim_{1}$ \\
\hline$\diamond_{1} p \Rightarrow \square_{1} p$ & $\sim_{1}=i d_{W}$ \\
\hline$\diamond_{1} p \Rightarrow \square_{2} p$ & $\sim_{1}=\sim_{2}=i d_{W}$ \\
\hline$\diamond_{1} p \Rightarrow \triangleright_{2} p$ & $\sim_{1} \subseteq \sim_{2}$ \\
\hline
\end{tabular}

Fig. 1. Proper extensions of $\mathrm{S}_{2}$ generated by axioms of the form $\square \phi \Rightarrow \boxminus \phi$. Formulas not included in the table but which are instances of this schema give completeness with respect to equivalence frames.

Theorem 2. An equivalence frame $F$ validates one of the axioms in Figure 1 if and only if $F$ has the corresponding property.

Theorem 3. All the logics $S 5_{2}+\{\phi\}$, where $\phi$ is a conjunction of formulas expressible from axiom schema 1 are sound and complete with respect to the intersection of the respective class of frames reported in Fig. 1.

The results of Theorem 3 are quite well known. The most important logic is probably the one that forces the knowledge of an agent to be a subset of the knowledge of another. In Section 1 we have discussed two scenarios in which this can be proven useful. Stronger logics can be defined by assuming that the modal component for one of the agents collapses onto the propositional calculus. When this happens we are in a situation in which "being possible according to one agent" is equivalent to "being known" and this in turn is equivalent to "being true". It is clear that this is indeed a very strong constraint which limits the expressivity of our language. Still these logics can be proven to be consistent.

The strongest consistent logic is Triv $_{2}^{2}$ that can be defined from $\mathrm{S}_{2}$ by adding the axiom $\searrow_{1} p \Rightarrow \square_{2} p$ to $\mathrm{S}_{2}$ or equivalently by adding both $\diamond_{1} p \Rightarrow \square_{1} p$ and $\searrow_{2} p \Rightarrow \square_{2} p$. In this logic the two agents have equal knowledge that is equivalent to the truth on the world of evaluation.

\section{Interaction axioms of the form $\square p \Rightarrow \boxminus \odot p$}

There are 64 axioms of the shape

$$
\sqcup \phi \Rightarrow \boxminus \boxminus \phi \text { where } \square \in\left\{\square_{1}, \square_{2}, \diamond_{1}, \diamond_{2}\right\} .
$$

Factoring 1-2 symmetries reduces this number to 32. Again, many of these (14 in number) do not generate proper extensions of $\mathrm{S}_{2}{ }^{3}$. For the remaining 18, the completeness results for the extension they generate are more complicated than the ones in the previous section. We present one result in detail (the reader is referred to [14] for the other proofs): it concerns the axiom

$$
\square_{1} p \Rightarrow \diamond_{1} \square_{2} p
$$

\footnotetext{
${ }^{2}$ Triv $_{2}$ is the logic obtained from $\mathrm{S}_{2}$ by adding $\square_{1} p \Leftrightarrow p$ and $\square_{2} p \Leftrightarrow p$.

${ }^{3} \square_{1} p \Rightarrow \diamond_{1} \square_{1} p, \square_{1} p \Rightarrow \diamond_{1} \diamond_{1} p, \square_{1} p \Rightarrow \diamond_{1} \diamond_{2} p, \square_{1} p \Rightarrow \square_{1} \square_{1} p, \square_{1} p \Rightarrow \square_{1} \diamond_{1} p, \square_{1} p \Rightarrow$ $\square_{1} \diamond_{2} p, \square_{1} p \Rightarrow \diamond_{2} \square_{1} p, \square_{1} p \Rightarrow \diamond_{2} \diamond_{1} p, \square_{1} p \Rightarrow \diamond_{2} \diamond_{2} p, \square_{1} p \Rightarrow \square_{2} \diamond_{2} p, \diamond_{2} p \Rightarrow \diamond_{1} \diamond_{2} p$, $\diamond_{2} p \Rightarrow \diamond_{2} \diamond_{1} p, \diamond_{2} p \Rightarrow \diamond_{2} \diamond_{2} p$, and $\diamond_{2} p \Rightarrow \square_{2} \diamond_{2} p$.
} 
Lemma 2. Let $F$ be an equivalence frame. $F \models \square_{1} p \Rightarrow \diamond_{1} \square_{2} p$ if and only if $F$ is such that $\forall w \exists w^{\prime} \in[w]_{\sim_{1}}:\left[w^{\prime}\right]_{\sim_{2}} \subseteq[w]_{\sim_{1}}$.

$\left([w]_{\sim_{1}}\right.$ is the $\sim_{1}$-equivalence class of $w$.)

Proof. From right to left; consider any model $M$ and a point $w$ in it such that $M \models_{w}$ $\square_{1} p$. So, for every point $w^{\prime}$ such that $w \sim_{1} w^{\prime}$ we have $M \models_{w^{\prime}} p$. But, by assumption, there exists a point $w^{\prime} \in[w]_{\sim_{1}}$ such that $\left[w^{\prime}\right]_{\sim_{2}} \subseteq[w]_{\sim_{1}}$. So, $p$ holds at any point of the equivalence class $\left[w^{\prime}\right]_{\sim_{2}}$, and so $M \models_{w^{\prime}} \square_{2} p$. Therefore $M \models_{w} \diamond_{1} \square_{2} p$.

For the converse, suppose the relational property above does not hold. Then there exists a frame $F$ and a point $w$ in $F$ such that for any $w^{\prime} \in[w]_{\sim_{1}}$ we have $\left[w^{\prime}\right]_{\sim_{2}} \not \subset$ $[w]_{\sim_{1}}$, i.e. we have the existence of a point $w^{\prime \prime} \in\left[w^{\prime}\right]_{\sim_{2}}$ such that $w^{\prime \prime} \notin[w]_{\sim_{1}}$. Consider a valuation $\pi$ such that $\pi(p)=\left\{w^{\prime} \mid w \sim_{1} w^{\prime}\right\}$. We have $(F, \pi) \models_{w} \square_{1} p$ and $(F, \pi) \not \forall_{w^{\prime \prime}} p$. So $(F, \pi) \not \forall_{w^{\prime}} \square_{2} p$. So we have $(F, \pi) \not \forall_{w} \diamond_{1} \square_{2} p$ which is absurd.

Lemma 3. The logic $S 5_{2}+\left\{\square_{1} p \Rightarrow \diamond_{1} \square_{2} p\right\}$ is sound and complete with respect to equivalence frames satisfying the property $\forall w \exists w^{\prime} \in[w]_{\sim_{1}}:\left[w^{\prime}\right]_{\sim_{2}} \subseteq[w]_{\sim_{1}}$.

Proof. Soundness was proven in first part of Lemma 2.

For completeness we prove that the logic $\mathrm{S}_{2}+\left\{\square_{1} p \Rightarrow \diamond_{1} \square_{2} p\right\}$ is canonical. In order to do that, suppose, by contradiction, that the frame of the canonical model does not satisfy the relational property above. Then, it must be that there exists a point $w$ such that:

$$
\forall w^{\prime} \in[w]_{\sim_{1}} \exists w^{\prime \prime}: w^{\prime} \sim_{2} w^{\prime \prime} \text { and } w \chi_{1} w^{\prime \prime} .
$$

Call $w_{1}^{\prime}, \ldots, w_{n}^{\prime}, \ldots$ the points in $[w]_{\sim_{1}}$, and $w_{i}^{\prime \prime}$ the point in $\left[w_{i}^{\prime}\right]_{\sim_{2}}$ such that $w \chi_{1}$ $w_{i}^{\prime \prime} ; i=1, \ldots, n, \ldots$. Recall (for example see [10], page 118) that $w \sim_{1} w^{\prime}$ on the canonical model is defined as $\forall \alpha \in \mathcal{L}\left(\square_{i} \alpha \in w\right.$ implies $\left.\alpha \in w^{\prime}\right) ; w \chi_{j} w^{\prime}$ is defined as $\exists \alpha \in \mathcal{L}\left(\square_{j} \alpha \in w\right.$ and $\left.\neg \alpha \in w^{\prime}\right)$. So we can find some formulas $\alpha_{i} \in$ $\mathcal{L} ; i=1, \ldots, n, \ldots$ such that $\square_{1} \alpha_{i} \in w, \alpha_{i} \in w_{i}^{\prime}, \neg \alpha_{i} \in w_{i}^{\prime \prime} ; i=1, \ldots, n, \ldots$ Call $\alpha=\wedge_{i=1}^{n} \alpha_{i}$; we have $\square_{1} \alpha_{i} \in w ; i=1, \ldots, n, \ldots$. So $\square_{1} \alpha \in w$. But $\neg \alpha \in$ $w_{i}^{\prime \prime}, i=1, \ldots, n, \ldots$. So $\searrow_{2} \alpha \in w_{i}^{\prime}$ for every $i$ in $\{1, \ldots, n, \ldots\}$. So $\square_{1} \searrow_{2} \neg \alpha \in w$, i.e. $\neg \diamond_{1} \square_{2} \alpha \in w$. But $\square_{1} \alpha \in w$ and $\vdash \square_{1} \alpha \Rightarrow \diamond_{1} \square_{2} \alpha$, so $w$ would be inconsistent. Therefore the canonical frame must satisfy the property above and the logic is complete with respect to equivalence frames satisfying the property $\forall w \exists w^{\prime} \in[w]_{\sim_{1}}:\left[w^{\prime}\right]_{\sim_{2}} \subseteq$ $[w]_{\sim_{1}}$.

Similar results hold for the other 17 axioms of the form 2, and the situation is summarised in Fig. 2. See [14] for full details.

Theorem 4. All the logics $S 5_{2}+\{\phi\}$, where $\phi$ is a conjunction of formulas expressible from axiom schema 2 are sound and complete with respect to the intersection of the respective class of frames reported in Fig. 2.

Among all these axioms, the most intuitive ones in terms of knowledge are probably $\square_{1} p \Rightarrow \square_{2} \square_{1} p$ and its "dual" $\square_{2} p \Rightarrow \square_{1} \square_{2} p$, in which one agent knows that the other knows something every time this happens to be the case. It is interesting to see that this is equivalent to one agent knowing everything known by the other agent. 


\begin{tabular}{|c|c|}
\hline Interaction Axioms & Completeness \\
\hline$\square_{1} p \Rightarrow \diamond_{1} \square_{2} p$ & $\forall w \exists w^{\prime} \in[w]_{\sim_{1}}:\left[w^{\prime}\right]_{\sim_{2}} \subseteq[w]_{\sim_{1}}$ \\
\hline$\square_{1} p \Rightarrow \square_{2} \square_{1} p$ & $\sim_{2} \subseteq \sim_{1}$ \\
\hline$\square_{1} p \Rightarrow \square_{2} \square_{2} p$ & $\sim_{2} \subseteq \sim_{1}$ \\
\hline$\square_{1} p \Rightarrow \square_{2} \searrow_{1} p$ & $\sim_{2} \subseteq \sim_{1}$ \\
\hline$\square_{1} p \Rightarrow \square_{1} \square_{2} p$ & $\sim_{2} \subseteq \sim_{1}$ \\
\hline$\square_{1} p \Rightarrow \diamond_{2} \square_{2} p$ & $\sim_{2} \subseteq \sim_{1}$ \\
\hline$\diamond_{1} p \Rightarrow \diamond_{1} \square_{1} p$ & $\sim_{1}=i d_{W}$ \\
\hline$\diamond_{1} p \Rightarrow \diamond_{1} \square_{2} p$ & $\sim_{2}=i d_{W}$ \\
\hline$\diamond_{1} p \Rightarrow \square_{1} \square_{1} p$ & $\sim_{1}=i d_{W}$ \\
\hline$\diamond_{1} p \Rightarrow \square_{1} \square_{2} p$ & $\sim_{1}=\sim_{2}=i d_{W}$ \\
\hline$\diamond_{1} p \Rightarrow \square_{1} \diamond_{2} p$ & $\sim_{1} \subseteq \sim_{2}$ \\
\hline$\diamond_{1} p \Rightarrow \diamond_{2} \square_{1} p$ & $\sim_{1}=i d_{W}$ \\
\hline$\diamond_{1} p \Rightarrow \diamond_{2} \square_{2} p$ & $\sim_{1}=\sim_{2}=i d_{W}$ \\
\hline$\diamond_{1} p \Rightarrow \diamond_{2} \diamond_{2} p$ & $\sim_{1} \subseteq \sim_{2}$ \\
\hline$\diamond_{1} p \Rightarrow \square_{2} \square_{1} p$ & $\sim_{2}=\sim_{1}=i d_{W}$ \\
\hline$\diamond_{1} p \Rightarrow \square_{2} \square_{2} p$ & $\sim_{1}=\sim_{2}=i d_{W}$ \\
\hline$\diamond_{1} p \Rightarrow \square_{2} \searrow_{1} p$ & $\sim_{2} \subseteq \sim_{1}$ \\
\hline$\diamond_{1} p \Rightarrow \square_{2} \searrow_{2} p$ & $\sim_{1} \subseteq \sim_{2}$ \\
\hline
\end{tabular}

Fig. 2. Proper extensions of $\mathrm{S}_{2}$ generated by axioms of the form $\square \phi \Rightarrow \square \square \phi$. Formulas not included in the table but which are instances of the schema give completeness with respect to equivalence frames.

A more subtle, independent axiom expressed by axiom schema 2 is the formula ${ }^{4}$ :

$$
\square_{1} p \Rightarrow \diamond_{1} \square_{2} p
$$

which reads "If agent 1 knows $p$, then he considers possible that agent 2 also knows $p$ ". The above is an axiom that regulates a natural kind of "prudence" assumption of agent 1 in terms of what knowledge agent 2 may have. This is meaningful in MAS in which agents have similar characteristics. In these scenarios when an agent knows a fact, it may be appropriate to assume that the other agent, by acquiring the same information from the environment and by following her same reasoning, could have reached the same conclusion. Note that very often humans act as if they followed this axiom.

We leave it to the reader to explore other interactions from the table above.

Note that by taking the contrapositive of axiom schema 2 we can express axioms of the form $\sqcup \sqcup p \Rightarrow \boxminus p$. So all those axioms are also covered in this section. For simplicity we do not report the case of antecedents indexed as 2, but by applying symmetry it is straightforward to generate the corresponding axioms.

\footnotetext{
${ }^{4}$ The technical details of this formula have been discussed in Lemma 2 and Lemma 3.
} 


\section{Interaction axioms of the form $\square \square p \Rightarrow \boxminus \square p$}

We now discuss the most complex class of axioms we will see in this paper, i.e. extensions of $\mathrm{S}_{2}$ with interaction axioms expressible as:

$$
\bullet \bullet \phi \Rightarrow \boxminus \bullet \phi \text {, where } \bullet \in\left\{\square_{1}, \square_{2}, \diamond_{1}, \diamond_{2}\right\} .
$$

Of the 256 such axioms, we lose half by 1-2 symmetry; of the remaining 128, 64 of them begin with $\square_{i} \square_{j}$ with $i=j$, which, by well known S5 equivalences (Lemma 1) collapse to a case of the previous section. The remaining 64 axioms divide into 26 which do not induce proper extensions of $\mathrm{S}_{2}{ }^{5}$ and 38 axioms which do. Figure 3 summarises the result for the proper extensions.

Some results for axioms of the form of axiom schema 3 are already available in the literature:

Lemma 4 ([2]). The logic $S 5_{2}+\left\{\diamond_{1} \square_{2} p \Rightarrow \square_{2} \diamond_{1} p\right\}$ is sound and complete with respect to frames satisfying the property: for all $w, w_{1}, w_{2} \in W$ such that $w \sim_{1} w_{1}$, $w \sim_{2} w_{2}$ there exists a point $\bar{w}$ such that $w_{1} \sim_{2} \bar{w}, w_{2} \sim_{1} \bar{w}$.

Figure 3 shows that many of the extensions are equivalent to some logic examined in the previous sections. For example, we have the following.

Lemma 5. The logic $S 5_{2}+\left\{\oslash_{1} \square_{2} p \Rightarrow \square_{1} \square_{2} p\right\}$ is sound and complete with respect to equivalence frames such that $\sim_{1} \subseteq \sim_{2}$.

Proof. We show that the logic S5 $5_{2}+\left\{\diamond_{1} \square_{2} p \Rightarrow \square_{1} \square_{2} p\right\}$ is equivalent to the logic $\mathrm{S}_{2}+\left\{\square_{2} p \Rightarrow \square_{1} p\right\}$. In order to see this, we prove that:

$$
\vdash_{S 5_{2}+\left\{\diamond_{1} \square_{2} p \Rightarrow \square_{1} \square_{2} p\right\}} \square_{2} p \Rightarrow \square_{1} p \text { and } \vdash_{S 5_{2}+\left\{\square_{2} p \Rightarrow \square_{1} p\right\}} \oslash_{1} \square_{2} p \Rightarrow \square_{1} \square_{2} p
$$

where $\mathrm{S}_{2+\phi}$ is the logic (closed under uniform substitution) obtained from $\mathrm{S} 5_{2}$ by adding the formula $\phi$.

From left to right. Suppose $\diamond_{1} \square_{2} p \Rightarrow \square_{1} \square_{2} p$. We have $\diamond_{1} p \Rightarrow \diamond_{1} \diamond_{2} p$ by T. But since, by contraposition of the hypothesis, we have $\diamond_{1} \diamond_{2} p \Rightarrow \square_{1} \diamond_{2} p$, we obtain $\diamond_{1} p \Rightarrow \square_{1} \diamond_{2} p$, which in turn implies $\diamond_{1} p \Rightarrow \diamond_{2} p$.

From right to left. Suppose $\diamond_{1} p \Rightarrow \diamond_{2} p$ and substitute $\square_{2} p$ for $p$ in it. We obtain $\diamond_{1} \square_{2} p \Rightarrow \diamond_{2} \square_{2} p$, which is equivalent to $\diamond_{1} \square_{2} p \Rightarrow \square_{2} p$. Now, by necessitating by $\square_{1}$ and distributing the box by using axiom $\mathrm{K}$, we obtain $\square_{1} \diamond_{1} \square_{2} p \Rightarrow \square_{1} \square_{2} p$, which, given Lemma 1 gives us to the result $\diamond_{1} \square_{2} p \Rightarrow \square_{1} \square_{2} p$.

Since each of the two formulas above can be proven from the other within $\mathrm{S}_{2}$, we have that any proof of a formula in one logic can be repeated in the other. Now, since $\mathrm{S}_{2}+\left\{\diamond_{1} p \Rightarrow \searrow_{2} p\right\}$ is complete (see Figure 1) with respect to equivalence frames such that $\sim_{1} \subseteq \sim_{2}$, then also $S 5_{2}+\left\{\diamond_{1} \square_{2} p \Rightarrow \square_{1} \square_{2} p\right\}$ is sound and complete with respect to the same class of frames.

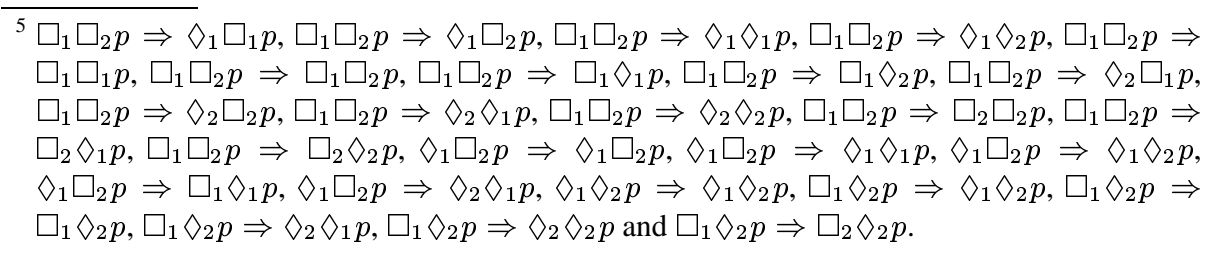




\begin{tabular}{|c|c|}
\hline Interaction Axioms & Completeness \\
\hline$\square_{1} \diamond_{2} p \Rightarrow \square_{1} \diamond_{1} p$ & $\forall w \exists w^{\prime} \in[w]_{\sim_{1}}:\left[w^{\prime}\right]_{\sim_{2}} \subseteq[w]_{\sim_{1}}$ \\
\hline$\square_{1} \diamond_{2} p \Rightarrow \diamond_{1} \diamond_{1} p$ & $\forall w \exists w^{\prime} \in[w]_{\sim_{1}}:\left[w^{\prime}\right]_{\sim_{2}} \subseteq[w]_{\sim_{1}}$ \\
\hline$\square_{1} \diamond_{2} p \Rightarrow \diamond_{1} \square_{2} p$ & $? \forall w \exists w^{\prime} \in[w]_{\sim_{1}}:\left[w^{\prime}\right]_{\sim_{2}}=\left\{w^{\prime}\right\}$ \\
\hline$\diamond_{1} \searrow_{2} p \Rightarrow \diamond_{2} \square_{1} p$ & $\sim_{1}=i d_{W}$ \\
\hline$\searrow_{1} \square_{2} p \Rightarrow \square_{1} \square_{1} p$ & $\sim_{1} \subseteq \sim_{2}$ \\
\hline$\diamond_{1} \square_{2} p \Rightarrow \diamond_{1} \square_{1} p$ & $\sim_{1} \subseteq \sim_{2}$ \\
\hline$\searrow_{1} \square_{2} p \Rightarrow \square_{1} \square_{2} p$ & $\sim_{1} \subseteq \sim_{2}$ \\
\hline$\diamond_{1} \square_{2} p \Rightarrow \square_{1} \diamond_{2} p$ & $\sim_{1} \subseteq \sim_{2}$ \\
\hline$\searrow_{1} \square_{2} p \Rightarrow \square_{2} \square_{1} p$ & $\sim_{1} \subseteq \sim_{2}$ \\
\hline$\diamond_{1} \square_{2} p \Rightarrow \square_{2} \square_{2} p$ & $\sim_{1} \subseteq \sim_{2}$ \\
\hline$\diamond_{1} \square_{2} p \Rightarrow \square_{2} \searrow_{2} p$ & $\sim_{1} \subseteq \sim_{2}$ \\
\hline$\diamond_{1} \square_{2} p \Rightarrow \diamond_{2} \square_{1} p$ & $\sim_{1} \subseteq \sim_{2}$ \\
\hline$\diamond_{1} \square_{2} p \Rightarrow \diamond_{2} \square_{2} p$ & $\sim_{1} \subseteq \sim_{2}$ \\
\hline$\diamond_{1} \square_{2} p \Rightarrow \diamond_{2} \searrow_{2} p$ & $\sim_{1} \subseteq \sim_{2}$ \\
\hline$\diamond_{1} \diamond_{2} p \Rightarrow \square_{1} \diamond_{2} p$ & $\sim_{1} \subseteq \sim_{2}$ \\
\hline$\diamond_{1} \diamond_{2} p \Rightarrow \square_{2} \searrow_{2} p$ & $\sim_{1} \subseteq \sim_{2}$ \\
\hline$\diamond_{1} \diamond_{2} p \Rightarrow \diamond_{2} \diamond_{2} p$ & $\sim_{1} \subseteq \sim_{2}$ \\
\hline$\diamond_{1} \diamond_{2} p \Rightarrow \square_{1} \square_{1} p$ & $\sim_{1}=\sim_{2}=i d_{W}$ \\
\hline$\diamond_{1} \diamond_{2} p \Rightarrow \square_{1} \square_{2} p$ & $\sim_{1}=\sim_{2}=i d_{W}$ \\
\hline$\diamond_{1} \diamond_{2} p \Rightarrow \square_{2} \square_{1} p$ & $\sim_{1}=\sim_{2}=i d_{W}$ \\
\hline$\diamond_{1} \diamond_{2} p \Rightarrow \square_{2} \square_{2} p$ & $\sim_{1}=\sim_{2}=i d_{W}$ \\
\hline$\diamond_{1} \diamond_{2} p \Rightarrow \diamond_{1} \square_{1} p$ & $\sim_{1}=\sim_{2}=i d_{W}$ \\
\hline$\diamond_{1} \diamond_{2} p \Rightarrow \diamond_{2} \square_{2} p$ & $\sim_{1}=\sim_{2}=i d_{W}$ \\
\hline$\square_{1} \diamond_{2} p \Rightarrow \square_{1} \square_{1} p$ & $\sim_{2}=i d_{W}$ \\
\hline$\square_{1} \diamond_{2} p \Rightarrow \diamond_{1} \square_{1} p$ & $\sim_{2}=i d_{W}$ \\
\hline$\square_{1} \diamond_{2} p \Rightarrow \square_{2} \diamond_{1} p$ & $\sim_{2} \subseteq \sim_{1}$ \\
\hline$\diamond_{1} \diamond_{2} p \Rightarrow \square_{1} \diamond_{1} p$ & $\sim_{2} \subseteq \sim_{1}$ \\
\hline$\diamond_{1} \diamond_{2} p \Rightarrow \square_{2} \diamond_{1} p$ & $\sim_{2} \subseteq \sim_{1}$ \\
\hline$\diamond_{1} \diamond_{2} p \Rightarrow \diamond_{1} \diamond_{1} p$ & $\sim_{2} \subseteq \sim_{1}$ \\
\hline$\square_{1} \diamond_{2} p \Rightarrow \square_{1} \square_{2} p$ & $\sim_{2}=i d_{W}$ \\
\hline$\square_{1} \diamond_{2} p \Rightarrow \square_{2} \square_{1} p$ & $\sim_{2}=i d_{W}$ \\
\hline$\square_{1} \diamond_{2} p \Rightarrow \square_{2} \square_{2} p$ & $\sim_{2}=i d_{W}$ \\
\hline$\square_{1} \diamond_{2} p \Rightarrow \diamond_{2} \square_{2} p$ & $\sim_{2}=i d_{W}$ \\
\hline$\diamond_{1} \diamond_{2} p \Rightarrow \diamond_{1} \square_{2} p$ & $\sim_{2}=i d_{W}$ \\
\hline$\square_{1} \square_{2} p \Rightarrow \square_{2} \square_{1} p$ & $\begin{array}{l}w \sim_{1} w_{1}, w \sim_{2} w_{2} \Rightarrow \exists \bar{w}: \\
w_{1} \sim_{2} \bar{w}, w_{2} \sim_{1} \bar{w}\end{array}$ \\
\hline$\diamond_{1} \diamond_{2} p \Rightarrow \diamond_{2} \diamond_{1} p$ & $\begin{array}{l}w \sim_{1} w_{1}, w \sim_{2} w_{2} \Rightarrow \exists \bar{w}: \\
w_{1} \sim_{2} \bar{w}, w_{2} \sim_{1} \bar{w}\end{array}$ \\
\hline$\diamond_{1} \square_{2} p \Rightarrow \square_{2} \diamond_{1} p$ & $\begin{array}{l}w \sim_{1} w_{1}, w \sim_{2} w_{2} \Rightarrow \exists \bar{w}: \\
w_{1} \sim_{2} \bar{w}, w_{2} \sim_{1} \bar{w}\end{array}$ \\
\hline$\square_{1} \diamond_{2} p \Rightarrow \diamond_{2} \square_{1} p$ & ? Either $\sim_{1}=i d_{W}$ or $\sim_{2}=i d_{W}$ \\
\hline
\end{tabular}

Fig. 3. Proper extensions of $\mathrm{S} 5_{2}$ generated by axioms of the form $\square \square \phi \Rightarrow \square \square \phi$. For axioms listed with "?" correspondence is proved but completeness is only conjectured. 
Axioms of shape 3 are intrinsically much harder (from a model-theoretic point of view) to examine with the basic tools than any other examined so far because they can express antecedents of the form $\square_{1} \diamond_{2}$. These axioms represent knowledge of agent 1 about facts considered possible by agent 2 . Technically, these formulas reminds us of the the McKinsey axiom of mono-modal logic, which has represented a challenging problem for logicians for many years and has been solved not too long ago by Goldblatt [5].

Consider axiom $\square_{1} \diamond_{2} p \Rightarrow \diamond_{1} \square_{2} p$. With this axiom we rule out situations in which agent 1 knows that $p$ is considered possible by agent 2 and agent 1 also knows that $\neg p$ is considered possible by agent 2 .

Definition 1. A point $w \in W$ is called an i-dead-end if for all $w^{\prime} \in W$ we have $w \sim_{i} w^{\prime}$ implies $w=w^{\prime}$.

Lemma 6. Given a frame $F=\left(W, \sim_{1}, \sim_{2}\right)$ and a point $w$ on it, $w$ is an $i$-dead-end if any only if for any valuation $\pi$, we have $(F, \pi) \models_{w} p \Rightarrow \square_{i} p$.

We can then prove the results for this axiom.

Lemma 7. $F \models \square_{1} \diamond_{2} p \Rightarrow \diamond_{1} \square_{2} p$ if and only if $F$ is such that every point $w$ is related by relation 1 to a 2-dead-end; i.e. for all $w \in W$ there exists a $w^{\prime} \in W, w \sim_{1} w^{\prime}$ such that $\left[w^{\prime}\right]_{\sim_{2}}=\left\{w^{\prime}\right\}$.

Proof. From right to left; consider any model $M$ such that every point sees via 1 a $2-$ dead-end. Suppose $M \models_{w} \square_{1} \diamond_{2} p$; so for every point $w^{\prime}$ such that $w \sim_{1} w^{\prime}$ we have that there must be a $w^{\prime \prime}$ such that $w^{\prime} \sim_{2} w^{\prime \prime}$ and $M \models{ }_{w^{\prime \prime}} p$. But by assumption one of the $w^{\prime}$ is a 2-dead-end, so we have the existence of a point $\bar{w} \in[w]_{\sim_{1}}$ such that (by Lemma 6) $M \models_{\bar{w}} \square_{2} p$. Then $M \models_{w} \diamond_{1} \square_{2} p$.

For the converse, consider any equivalence frame $F$, such that $F \models \square_{1} \diamond_{2} p \Rightarrow$ $\diamond_{1} \square_{2} p$ and suppose by contradiction that the property above does not hold. Consider the set $X=[w]_{\sim_{1}}$, the equivalence relation $\sim=\sim_{1} \cap \sim_{2}$ and the quotient set $X / \sim$. Consider now the set $Y$ constructed by taking one and only one representative $w$ for each class $[w]_{\sim}$ in $X / \sim$. Consider a valuation $\pi(p)=Y$ and consider the model $M=$ $\left(W, \sim_{1}, \sim_{2}, \pi\right)$. By construction we have $M \models_{w} \square_{1} \diamond_{2} p$. Then by our assumption we also have $M \models_{w} \diamond_{1} \square_{2} p$. So there must be a point $w^{\prime}$ such that $w \sim_{1} w^{\prime}$ such that $M \models w^{\prime} \square_{2} p$. But since $w^{\prime}$ by assumption is not a 2 -dead-end, the equivalence class $\left[w^{\prime}\right]_{\sim_{2}}$ must contain more than $w^{\prime}$ itself and by construction $p$ is true only at one point in that class and false for every $y \notin X$. So we have $M \not \forall_{w^{\prime}} \square_{2} p$ for every $w^{\prime} \in[w]_{\sim_{1}}$ and so $M \forall_{w} \diamond_{1} \square_{2} p$, which is absurd. So for every point $w \in W$ there must be a 2-dead-end accessible from it.

Completeness for the above remains an open problem.

Conjecture 1. The logic S $5_{2}+\left\{\square_{1} \diamond_{2} p \Rightarrow \diamond_{1} \square_{2} p\right\}$ is sound and complete with respect to equivalence frames such that every point is related by relation 1 to a 2-dead-end; i.e. for all $w \in W$ there exists a $w^{\prime} \in W, w \sim_{1} w^{\prime}$ such that $\left[w^{\prime}\right]_{\sim_{2}}=\left\{w^{\prime}\right\}$.

The same happens for the axiom $\square_{1} \diamond_{2} p \Rightarrow \diamond_{2} \square_{1} p$. This axiom represents the situation in which it cannot be that agent 1 knows that agent 2 considers possible $p$ while agent 2 knows that agent 1 considers possible $\neg p$. 
Lemma 8. $F \models \square_{1} \diamond_{2} p \Rightarrow \diamond_{2} \square_{1} p$ if and only $F$ is such that if in every connected sub-frame either $\sim_{1}=i d_{W}$ or $\sim_{2}=i d_{W}$.

Proof. From left to right. This part of the proof is structured as follows:

1. We prove that $F \models \square_{1} \diamond_{2} p \Rightarrow \diamond_{2} \square_{1} p$ implies that any point $w \in W$ either sees via 1 a 2 -dead-end, or the point $w$ sees via 2 a 1-dead-end.

2. We prove that if on a frame $F$ such that $F \models \square_{1} \diamond_{2} p \Rightarrow \diamond_{2} \square_{1} p$ and there is point $w$ which is an $i$-dead-end, then $\sim_{i}=i d_{W}$ on the whole connected sub-frame generated by $w$; where $i \in\{1,2\}$.

3. The two facts above together prove that if $F \models \square_{1} \diamond_{2} p \Rightarrow \diamond_{2} \square_{1} p$, then in every connected sub-frame either $\sim_{1}=i d_{W}$ or $\sim_{2}=i d_{W}$.

1) By contradiction, consider any connected equivalence frame $F$, in which a $w \in W$ does not see via $i$ any $j$-dead end, i.e. $\forall w^{\prime} \in[w]_{\sim_{i}},\left[w^{\prime}\right]_{\sim_{j}} \neq\left\{w^{\prime}\right\}, i \neq j, i, j \in\{1,2\}$; we prove that $F \not \forall \square_{1} \diamond_{2} p \Rightarrow \diamond_{2} \square_{1} p$. To see this, consider the set $X=[w]_{\sim_{1}} \cup[w]_{\sim_{2}} \backslash$ $\{w\}$, the equivalence relation $\sim=\sim_{1} \cap \sim_{2}$ and the quotient set $X / \sim$. Consider now the set $Y$ defined by taking one representative $y$ for every equivalence class $[y]_{\sim} \in X / \sim$ : the set $Y$ is such that $\forall y_{1}, y_{2} \in Y$ we have $\left[y_{1}\right]_{\sim} \cap\left[y_{2}\right]_{\sim}=\emptyset$ and $\bigcup_{y \in Y}[y]_{\sim}=$ $X$. Consider now the model $M=(F, \pi)$, by taking the valuation $\pi(p)=Y$. By construction, in the model $M$ for any $x \in X$, there is a point accessible from $x$ via $\sim_{2}$ which satisfies $p$, and since by hypothesis $w$ is neither a 1-dead-end nor a 2-dead-end (as otherwise it would see itself as dead-end) we have $M \models_{w} \square_{1} \triangleright_{2} p$. So by the validity of the axiom we also have $M \models_{w} \diamond_{2} \square_{1} p$, i.e. there must be a $w^{\prime} \in[w]_{\sim_{2}}$, such that $M \models w_{w^{\prime}} \square_{1} p$, but this is impossible because by hypothesis $\left[w^{\prime}\right]_{\sim_{1}} \neq\left\{w^{\prime}\right\}$, and by construction $p$ is true at just one point in $\left[w^{\prime}\right]_{\sim_{1}} \cap\left[w^{\prime}\right]_{\sim_{2}}$, and false at every point not in $X$. See Figure 4.

2) Consider now a connected frame $F$ such that $F \models \square_{1} \diamond_{2} p \Rightarrow \diamond_{2} \square_{1} p$ and suppose for example that $w$ is a 1-dead-end, we want to prove that $\sim_{1}=i d_{W}$ on the connected sub-frame generated by $w^{6}$. If $w$ is also a 2-dead-end, then $\sim_{1}=\sim_{2}=i d_{W}$ on the generated frame which gives us the result. If not, suppose that $\sim_{1} \neq i d_{W}$; so there must be two points $w^{\prime}, w^{\prime \prime} \in W ; w^{\prime} \neq w^{\prime \prime}$, such that $w^{\prime} \sim_{1} w^{\prime \prime}$. So, since the frame is connected, without loss of generality assume $w \sim_{2} w^{\prime}$. Consider now valuation $\pi(p)=\left\{x \mid x \in[w]_{\sim_{2}}, x \neq w^{\prime}\right\} \cup\left\{w^{\prime \prime}\right\}$ and the model $M=(F, \pi)$ built on $F$ from $\pi$. So, we have $M \models_{w} \square_{2} \diamond_{1} p$, and so, by validity of the axiom, we also have $M \models_{w} \diamond_{1} \square_{2} p$. So we must have $M \models_{w} \square_{2} p$, which is a contradiction because $M \models_{w^{\prime}} \neg p$.

So we have that if the axiom is valid, then in every connected component one of the two relations is the identity.

From right to left. Consider any equivalence model $M$ whose underlying frame satisfies the property above and suppose that $M \models_{w} \square_{1} \diamond_{2} p$.

Suppose $\sim_{1}=i d_{W}$ and $M \models_{w} \square_{1} \diamond_{2} p$, so there is a $w^{\prime} \in[w]_{\sim_{2}}$, such that $M \models_{w^{\prime}}$ $p$. But since $\sim_{1}=i d_{W}$ on the connected part, we also have $M \models_{w^{\prime}} \square_{1} p$. So $M \models_{w^{\prime}}$ $\diamond_{2} \square_{1} p$. Suppose now $\sim_{2}=i d_{W}$ and $M \models_{w} \square_{1} \diamond_{2} p$. So for every $w^{\prime} \in[w]_{\sim_{1}}$ we have $M \models_{w^{\prime}} p$. But then we also have $M \models_{w} \diamond_{2} \square_{1} p$.

\footnotetext{
${ }^{6}$ If $w$ is a 2-dead-end then the argument is symmetric.
} 


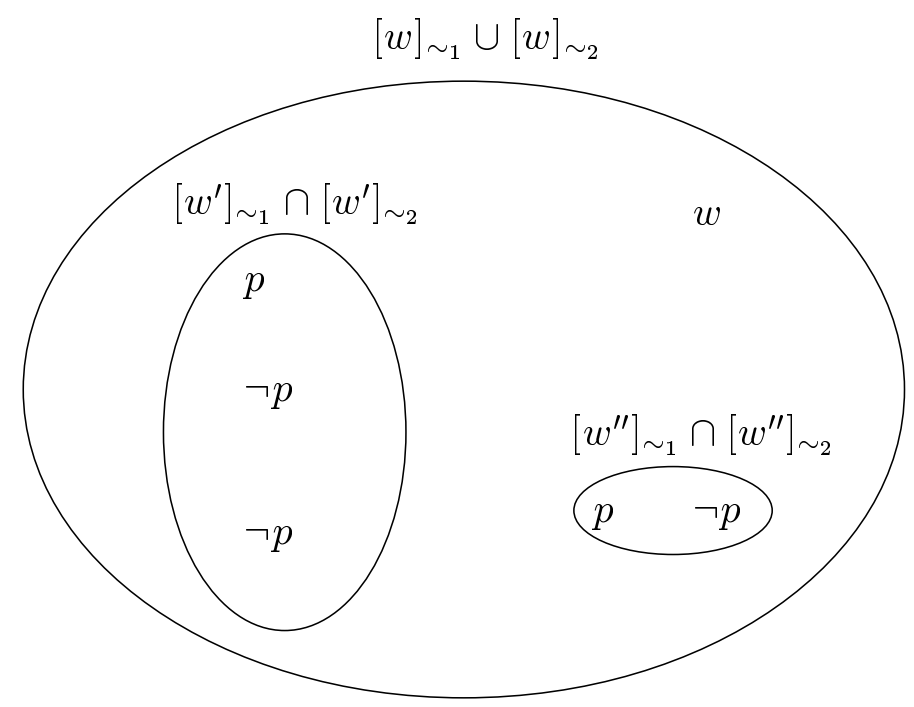

Fig. 4. A model not satisfying the property of Lemma 8 . Note that $[w]_{\sim_{1}} \neq\{w\}$ and $[w]_{\sim_{2}} \neq$ $\{w\}$.

Again we can only conjecture completeness with respect to the above class of frames.

Conjecture 2. The logic S5 $5_{2}+\left\{\square_{1} \searrow_{2} p \Rightarrow \diamond_{2} \square_{1} p\right\}$ is sound and complete with respect to equivalence frames such that either $\sim_{1}=i d_{W}$ or $\sim_{2}=i d_{W}$ on every connected subframe.

\section{Conclusions}

We have identified a number of non-trivial single-axiom extensions of $\mathrm{S}_{2}$ which specify a mode of interaction between two agents, and proved correspondence, soundness and completeness with respect to the appropriate classes of frames. The main contribution of this paper lies in the identification of a spectrum of interactions above $\mathrm{S}_{2}$.

Figure 5 represents graphically all the logics discussed so far together with the corresponding semantic classes (the ones for which we only conjectured completeness are not included). In the figure, the logics are ordered strength-wise. So, the strongest logic is of course $\operatorname{Triv}_{2}$ (represented as $\mathbf{S} 5_{2}+\left\{\diamond_{1} p \Rightarrow \square_{2} p\right\}$ ), the weakest simply $\mathbf{S} 5_{2}$. In between we have a few logic systems, the weakest of which are Catach's logic, and the two axioms that we examined in Theorem 2 and Theorem 3. Note that these three logics are independent. Stronger extensions include logics in which the knowledge of an agent is included in the knowledge of the other and combination of these. 


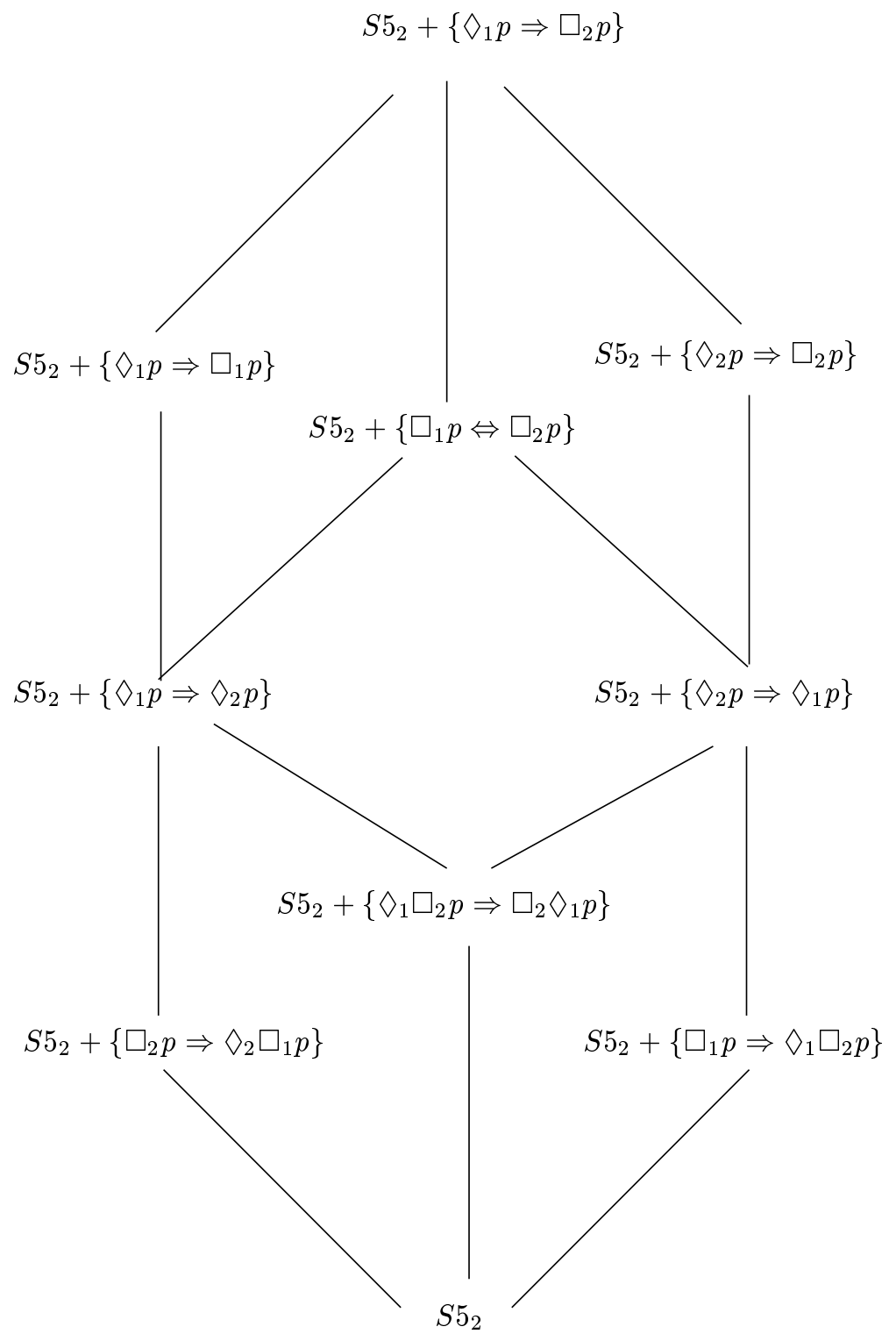

Fig. 5. The independent extensions of $\mathrm{S}_{2}$ that can be obtained by adding axioms of the shapes $\square \phi \Rightarrow \boxminus \phi$ and $\square \phi \Rightarrow \nabla \nabla \phi$ and the corresponding classes of frames. Formulas not included in the table but obtainable from the schema above give completeness with respect to equivalence frames. The logics for which results are only conjectured are not included in the figure. 
The fairly exhaustive analysis carried out in this paper permits the AI-user with an interaction axiom in mind to refer to the above tables to identify the class of Kripke frames that gives completeness. For most of the logics above decidability also follows because most of them have the finite model property.

We have given conjectures about the two McKinsey axioms (Conjecture 1, Conjecture 2). In private correspondence Van der Hoek [9] has communicated a proof of Conjecture 1, but the other axiom is still an open problem at this stage. Solving this issue is part of our future work.

The results presented in this paper conceptually belong to a family of works in which the relation between different modalities in a single- or multi-agent setting is explored. Among the many in the literature that deal with the interaction between different internal mental states, we would like to cite [8] in which van der Hoek, building upon a previous work [12] by Kraus and Lehmann, extensively explores the relation between knowledge and belief. Turning to the relation between agent and environment, in this proceedings Wooldridge and Lomuscio capture the relation between visibility, perception, and knowledge by means of formal tools very similar to ones presented in these pages [19]. Although the logics presented in those works aim at capturing static properties, an interesting line of research concerns designing algorithms defined on Kripke structures that model the evolution of internal mental states $[6,16,11]$.

\section{References}

1. M. Baldoni. Normal Multimodal Logics: Automatic Deduction and Logic Programming Extension. PhD thesis, Dipartimento di Informatica, Universitá degli Studi di Torino, Italy, 1998.

2. L. Catach. Normal multimodal logics. In T. M. Smith and G. R. Mitchell, editor, Proceedings of the 7th National Conference on Artificial Intelligence (AAAI'88), pages 491-495, St. Paul, MN, August 1988. Morgan Kaufmann.

3. R. López de Mantaras, J. Amat, F. Esteva, M. López, and C. Sierra. Generation of unknown environment maps by cooperative low-cost robots. In W. L. Johnson and B. Hayes-Roth, editors, Proceedings of the 1st International Conference on Autonomous Agents, pages 164169, New York, February5-8 1997. ACM Press.

4. R. Fagin, J. Y. Halpern, Y. Moses, and M. Y. Vardi. Reasoning about Knowledge. MIT Press, Cambridge, 1995.

5. R. Goldblatt. The McKinsey axiom is not canonical. The Journal of Symbolic Logic, 56(2):554-562, June 1991.

6. J. Halpern and M. Vardi. Model checking vs. theorem proving: a manifesto, pages 151-176. Artificial Intelligence and Mathematical Theory of Computation. Academic Press, Inc, 1991.

7. J. Hintikka. Knowledge and Belief, an introduction to the logic of the two notions. Cornell University Press, Ithaca (NY) and London, 1962.

8. W. van der Hoek. Sytems for knowledge and belief. Journal of Logic and Computation, 3(2):173-195, April 1993.

9. W. van der Hoek. A note on the logic $\mathrm{S} 5_{n}+\left\{\diamond_{1} \square_{2} p \Rightarrow \square_{1} \diamond_{2} p\right\}$, 1999. Private Communication.

10. G. E. Hughes and M. J. Cresswell. A New Introduction to Modal Logic. Routledge, New York, 1996. 
11. I. Isozaki and H. Katsuno. Observability-based nested belief computation for multiagent systems and its formalization. In N.R. Jennings and Y. Lespérance, editors, Intelligent Agents VI - Proceedings of the Sixth International Workshop on Agent Theories, Architectures, and Languages (ATAL-99), Lecture Notes in Artificial Intelligence. Springer-Verlag, Berlin, 2000. In this volume.

12. S. Kraus and D. J. Lehmann. Knowledge, belief, and time. Theoretical Computer Science, 58:155-174, 1988.

13. S. A. Kripke. Semantic analysis of modal logic (abstract). Journal of Symbolic Logic, 24:323-324, 1959.

14. A. Lomuscio. Knowledge Sharing among Ideal Agents. PhD thesis, School of Computer Science, University of Birmingham, Birmingham, UK, June 1999.

15. A. Lomuscio and M. Ryan. Ideal agents sharing (some!) knowledge. In H. Prade, editor, Proceedings of Proceedings of the 13th European Conference on Artificial Intelligence, pages 557-561, August 1998.

16. A. Lomuscio and M. Ryan. An algorithmic approach to knowledge evolution. Artificial Intelligence for Engineering Design, Analysis and Manufacturing (AIEDAM), 13(2), 1999.

17. J.-J. Ch. Meyer and W. van der Hoek. Epistemic Logic for AI and Computer Science, volume 41 of Cambridge Tracts in Theoretical Computer Science. Cambdridge University Press, 1995.

18. S. Popkorn. First Steps in Modal Logic. Cambridge University Press: Cambridge, England, 1994.

19. M. Wooldridge and A. Lomuscio. Reasoning about visibility, perception and knowledge. In N.R. Jennings and Y. Lespérance, editors, Intelligent Agents VI - Proceedings of the Sixth International Workshop on Agent Theories, Architectures, and Languages (ATAL-99), Lecture Notes in Artificial Intelligence. Springer-Verlag, Berlin, 2000. In this volume. 